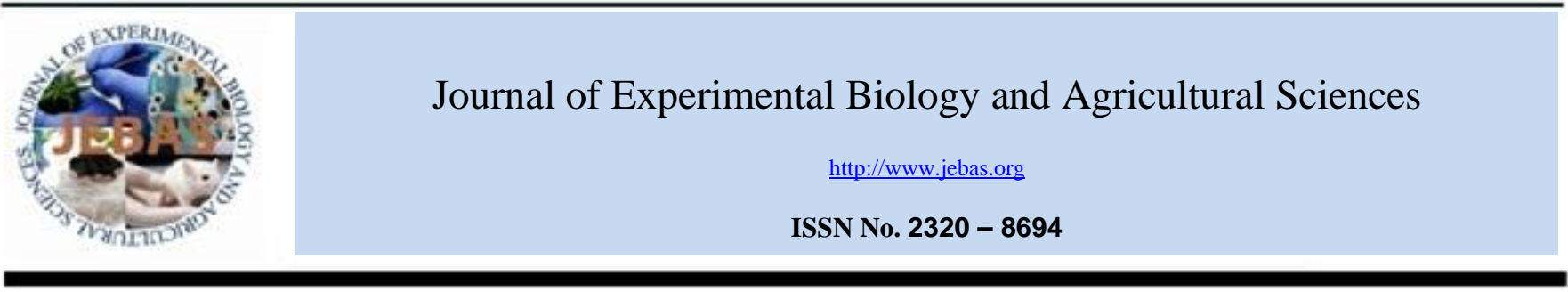

\title{
POTASSIUM SCHOENITE: AN EMERGING SOURCE OF POTASSIUM FOR IMPROVING GROWTH, YIELD AND QUALITY OF POTATO
}

\section{Dibakar Ghosh ${ }^{1,2}$, Sukamal Sarkar ${ }^{1, *}$, Koushik Brahmachari ${ }^{1}$, Sourav Garai ${ }^{1}$, Mriganka Pal $^{1}$ and Ashis Sharma ${ }^{3}$}

${ }^{1}$ Department of Agronomy, Bidhan Chandra Krishi Viswavidyalaya, Mohanpur, Nadia, West Bengal, India, 741252

${ }^{2}$ ICAR-Directorate of Weed Research, Jabalpur, Madhya Pradesh, India, 482004

${ }^{3}$ Agrocel Industries Pvt. Ltd. (Marine Chemicals Division), Goregaon (E), Mumbai, India, 400063

Received - January 23, 2017; Revision - March 22, 2017; Accepted - April 23, 2017

Available Online - May 12, 2017

DOI: http://dx.doi.org/10.18006/2017.5(2).173.182

KEYWORDS
Potassium schoenite
Foliar Spray
Potato
Yield
Quality

\begin{abstract}
A field experiment with potato (Solanum tuberosum L.) crop was carried out on an alluvial soil in West Bengal, India with the focal aim of assessing the relative effect of soil and foliar application of indigenously produced potassium schoenite (PS) in relation to that of muriate of potash (MOP) during winter seasons of 2014-15 and 2015-16 using a randomized complete-block design. The following treatment factors were evaluated: two sources of K (MOP and PS), two doses of K (100 and 75\% of recommended $\mathrm{K}$ as basal) and foliar application of PS (without and with). Experimental findings showed that different sources, doses and foliar application of PS significantly altered plant growth, yield and quality parameters (ascorbic acid, specific gravity and reducing sugar). Regarding, combination of different factors recommended doses of NPK (either PS or MOP as source of K) along with foliar PS fertilization was the best combination that resulted in the highest plant growth (height, dry matter accumulation), tuber number and yield of potato with respect to rest of the fertilization treatments. The same treatment combination exerted best potato quality parameters like ascorbic acid content, specific gravity and reducing sugar content. So, for getting higher yield and quality produce from potato, PS may be a good option for the potato-growers.
\end{abstract}

* Corresponding author

E-mail: sukamalsarkarc@yahoo.com (Sukamal Sarkar)

Peer review under responsibility of Journal of Experimental Biology and Agricultural Sciences.

Production and Hosting by Horizon Publisher India [HPI] (http://www.horizonpublisherindia.in/).

All rights reserved.
All the article published by Journal of Experimental Biology and Agricultural Sciences is licensed under a Creative Commons Attribution-NonCommercial 4.0 International License Based on a work at www.jebas.org.

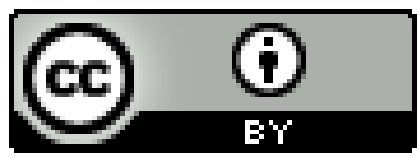




\section{Introduction}

Potato (Solanum tuberosum L.) is one of the most important crops grown both in sub-tropical and temperate regions of the world. It is the only crop having the potential like rice, wheat and maize to supplement the need for foods, of any nation (Alva et al., 2011). Potatoes are usually used for a wide range of table and processed foods, feeds and also for industrial purposes. India is the largest potato producer among the south-west Asian countries (Kadian et al., 2007) and globally ranks second after China in total potato production (FAO, 2014). Producing $12 \mathrm{mt}$ of potato from $0.41 \mathrm{~m}$ ha with a productivity of $29.7 \mathrm{tha}^{-1}$ West Bengal, amongst all the potato growing states of India, holds the second position nationally (Horticulture Statistics Division, GOI, 2014).

Potato requires a numbers of macro and micro plant nutrients for its growth and development. Nitrogen $(\mathrm{N})$, phosphorus $(\mathrm{P})$ and potassium $(\mathrm{K})$ are the most essential plant nutrients that are largely required by potato (Banerjee et al., 2016a). Being a strong Kloving crop, potato mines $\mathrm{K}$ in larger amounts than it consumes $\mathrm{N}$ and P (Panagiotopoulos, 1995). Further, Moinuddin et al. (2004) reported that for producing $29 \mathrm{t} \mathrm{ha}^{-1}$ of tubers, potato removed 91 $\mathrm{kg} \mathrm{K}_{2} \mathrm{O} \mathrm{ha}{ }^{-1}$. $\mathrm{K}$ is essentially required for the synthesis of sugars and starch and also for translocation of carbohydrates; it also plays an important role in maintaining growth and vigor of the plants (Singh et al., 1996; Banerjee et al., 2016b). Potassium is also responsible for higher yield and quality tuber production (Alva et al., 2011). Being a vegetative part of the plant, potato clicks with more nutrient inputs yielding more tubers; encouraging the farmers of the studied area to apply more and more chemical fertilizer to have more tuber yield without thinking of the tuber quality, soil health and environmental safety as a whole. Though the most widely used form of $\mathrm{K}$ fertilizer is $\mathrm{K}$-chloride $(\mathrm{KCl})$ in potato cultivation, Kumar et al. (2007) have reported that chloride form of $\mathrm{K}$ fertilization in potato above the recommended dose impacts drymatter percentage negatively, with reduced processing quality. Sulfate form of $\mathrm{K}$ fertilizers usually results in a higher dry-matter percentage in potato tubers than equivalent quantities of $\mathrm{K}$ in the chloride form (Smith, 1987); but it is less used by the potato growers because of its limited availability and relatively high price.

Indian potato fertilization system is mainly based upon $\mathrm{N}, \mathrm{P}$ and $\mathrm{K}$. Among them for $\mathrm{K}$ fertilizers, mainly muriate of potash (MOP) is completely dependent on import. Every year India has to spend a bulk of foreign exchange for importing potassium fertilizers. The country imported 3797 thousand tons of $\mathrm{K}$ fertilizer during 2014-15 (Department of Fertilizer, GOI, 2016). Indigenously, extraction process of sulphate of potash $\left(\mathrm{K}_{2} \mathrm{SO}_{4}\right)$ and schoenite $\left(\mathrm{K}_{2} \mathrm{SO}_{4}, \mathrm{MgSO}_{4}\right)$ from sea bittern was developed by Central Salt and Marine Chemicals Research Institute, Bhavnagar, Gujarat (Rathore et al., 2014) and some private agri-input agencies. Among them, indigenously produced double salts of $\mathrm{K}$, i.e., potassium schoenite (molar mass $415 \mathrm{~g} / \mathrm{mol}$ ) have been found to be beneficial on acidic and alluvial soils which provides a readily available supply of Potassium, Magnesium and Sulfur to growing plants in an ideal ratio.; although very little work has been reported so far (Sharma et al., 1998). Therefore, keeping the above facts in background, relative effect of soil and foliar application of indigenously produced potassium schoeniteas compared to that of MOP on potato were studied in the present investigation.

\section{Materials and Methods}

2.1 Climatic Conditions of the Study area

The field trial was carried out at District Seed Farm-C Unit, Bidhan Chandra Krishi Viswavidyalaya, Kalyani, West Bengal, India ( $22^{\circ} 58^{\prime} \mathrm{N}$ latitude, $88^{\circ} 25^{\prime} \mathrm{E}$ longitude, $8.5 \mathrm{~m}$ above mean sea level) during the winter season of 2014-15 and 2015-16.In the studied area the respective maximum and minimum temperature fluctuated between $37.3-24.8^{\circ} \mathrm{C}$ and $20.3-9.6^{\circ} \mathrm{C}$ in $2014-15$, and $35.1-$ $23.7^{\circ} \mathrm{C}$ and $21.8-9.3^{\circ} \mathrm{C}$ in $2015-16$. The maximum and minimum relative humidity of the experimental area prevailed between 89 and $34 \%$ in $2014-15$ and 97 and $34 \%$ in 2015- 16 respectively. The rainfall during the experimental period was 24.2 (5 rainy days) and $112.3 \mathrm{~mm}$ (14 rainy days) in both of the experimental year, respectively. It is evident from the above data that weather conditions were quite congenial for growth and development of potato crop during both the years of experimentation. The soil of study area was, sandy clay loam (56.5\% sand, $24.7 \%$ silt and 21.6 $\%$ clay) type with the following key properties for the $0-30 \mathrm{~cm}$ layer: pH 6.76 (in 1:2.5:: Soil : Water), EC $0.25 \mathrm{dS} \mathrm{m}^{-1}$ (in 1:2.5:: Soil : Water), organic carbon $0.57 \%$ (Wet oxidation method), available $\mathrm{N} 82.1 \mathrm{~kg} \mathrm{ha}^{-1}$ (Hot alkaline $\mathrm{KMnO}_{4}$ Method), available $\mathrm{P} 18.81 \mathrm{~kg} \mathrm{ha}^{-1}\left(0.5 \mathrm{M} \mathrm{NaHCO}_{3}\right.$ extract) and available $\mathrm{K} 167.3 \mathrm{~kg}$ $\mathrm{ha}^{-1}$ (Neutral $\mathrm{N} \mathrm{NH}_{4} \mathrm{OAc}$ extract).

\subsection{Treatments and experimental design}

The experiment was designed in randomized complete block with three factors being two sources of $\mathrm{K} v i z$. MOP and potassium schoenite (PS), two doses of K (100 and $75 \%$ of recommended K as basal) and foliar application of PS (without and with). The total treatment combinations were eight replicated thrice. Individual plot size for each treatment was $6 \mathrm{~m} \times 5 \mathrm{~m}$. Seed tubers of cv. Kufri Jyoti, a medium maturing (90-100 days) high yielding potato variety popularly grown in West Bengal, weighing about 30-40 g each were hand planted at a depth of $15 \mathrm{~cm}$ on 21 and 24 November of 2014 and 2015 respectively in tyne-made furrows at a spacing of $60 \mathrm{~cm} \times 20 \mathrm{~cm}$, and finally covered with soil. A uniform recommended dose (RD) of N-P i.e. 200-150 kg.ha-1 was applied in all the plots through urea [50\% before sowing and $50 \%$ at 30 days after planting (DAP)] and single super phosphate (100 $\%$ before sowing). Both the potassic fertilizers were applied along with $\mathrm{NK}$ fertilizer, as per treatment combinations. As foliar application $1.0 \%$ PS solution was applied at 30 and 50 DAP according to the treatment combination by a knapsack sprayer with a spray volume of $16 \mathrm{~L}$ capacity; the volume of water being $500 \mathrm{~L}$ $\mathrm{ha}^{-1}$. For the good germination and establishment of the crop two light irrigations were applied at 7 days interval within 12 DAP. Six more irrigations were given at an interval of $7-8$ days. For the purpose of weed management, metribuzin at $750 \mathrm{~g} \mathrm{ha}^{-1}$ was applied as pre-emergence at $3 \mathrm{DAP}$, and subsequent weed flushes were managed through manual measures in both the years of study. Earthing up was done at $30 \mathrm{DAP}$ and ridges were made manually with spade. 
No major insect pests and disease were observed during the cropping season in both of the experimental years. To check aphid infestation at later stages of crop growth dehaulming (removal of above ground biomass i.e. haulms) by sickle was performed at 15 days before harvesting of tubers. Potato was harvested on 23 and 24 February in 2015 and 2016, respectively.

\subsection{Plant sampling and observations}

In each plot, the second rows on either side were marked for destructive sampling vis-a-vis for recording other biometric observations and the middle four rows were marked for determination of tuber yield. To determine the germination percentage $(\%)$ the plants germinated from tubers was randomly counted at 30 DAP from five rows of each plot and expressed in percentage $(\%)$. Biometric observations viz. plant height, number of haulms hill ${ }^{-1}$ and number of compound leaves plant ${ }^{-1}$ are taken from the five randomly selected plants from each plots at 30, 50, 70 and 90 DAP. Leaf area index (LAI) for each treatment was calculated at 30, 50, 70 and 90 DAP by using the following formula (Watson, 1947).

$$
\text { LAI }=\frac{\text { Area of total number of leaves }}{\text { Ground area of sampled plants }}
$$

For recording dry weight, plants from the penultimate row in each plot were uprooted carefully and then sun-dried and oven dried $\left(70^{\circ} \mathrm{C}\right)$ to obtain a constant dry weight, and finally expressed in $\mathrm{g}$ plant $^{-1}$. Crop growth rate (CGR) was computed using following formula (Gardner, 1985):

$$
\mathrm{CGR}=\frac{\mathrm{W}_{2}-\mathrm{W}_{1}}{\mathrm{t}_{2}-\mathrm{t}_{1}} \times \mathrm{GAg} / \mathrm{m}^{2} / \text { day }
$$

Where, $\mathrm{W}_{2}$ and $\mathrm{W}_{1}$ are the final and initial dry weights of plant material per unit area at times $t_{2}$ (final time) and $t_{1}$ (initial time) respectively and GA is the ground area $\left(\mathrm{m}^{2}\right)$.

Tuber bulking rate (TBR) was calculated as described by Mozumder et al. (2014).

$$
\mathrm{TBR}=\frac{\mathrm{W}_{2}-\mathrm{W}_{1}}{\mathrm{t}_{2}-\mathrm{t}_{1}} \times \mathrm{GAg} / \mathrm{m}^{2} / \text { day }
$$

Where, $\mathrm{W}_{2}$ and $\mathrm{W}_{1}$ are the final and initial fresh weights of tuber per unit area at times $t_{2}$ (final time) and $t_{1}$ (initial time) respectively and GA is the ground area $\left(\mathrm{m}^{2}\right)$.

To determine the tuber yield and number five rows of $4 \mathrm{~m}$ length in the centre of each treatment plot was taken and finally represented in $\mathrm{tha}^{-1}$.

2.4 Determination of biochemical parameters
After harvesting of potato tubers, some biochemical analysis were done to judge the quality parameters as influenced by different sources, doses and methods of application of potassium fertilizers. Ascorbic acid content of potato was estimated by using 2, 6 di-chlorophenolindophenol dye titration method (Casanas et al., 2002). The reducing sugar (dry weight basis) of potato tuber was determined by following the method of Nelson (1944). Specific weight of potato was determined by hydrometer method using a sample of $3.63 \mathrm{~kg}$ of processing grade tubers from each plot (Gould, 1999).

\subsection{Statistical analysis}

The statistical analysis of data was done using SAS Windows Version 9.3 applying analysis of variance (PROC GLM) with subsequent multiple comparisons of means for both of the experimental years. The effect of the years was non-significant and there were no significant interactions between treatments and years. Therefore, the data were combined over the years and subjected to ANOVA. Treatment means were separated with the use of Tukey's Honestly Significant Difference test at the 5\% level of significance.

\section{Results}

\subsection{Potato Growth traits}

Plant germination recorded at 30 DAP revealed that neither the source nor the dose of $\mathrm{K}$ fertilization affected the final plant stand (Table 1); even combination of different factors recorded nonsignificant response to germination (Table 2). Source and dose of $\mathrm{K}$ fertilization significantly $(p \leq 0.05)$ influenced plant height of the crop at later growth stages (50, 70 and $90 \mathrm{DAP})$. Basal application of PS improved plant height significantly $(p \leq 0.05)$ than the MOP did (Table 1). A reduction in the dose $\mathrm{K}$ fertilizer $\left(\mathrm{RD}_{\mathrm{NP}} 3 / 4 \mathrm{~K}\right)$ resulted in a reduced plant height at 50 and 70 DAP by 6.1 and $6.3 \%$ respectively with respect to the crop fertilized with $\mathrm{RD}_{\mathrm{NPK}}$. On other hand, the plant height of potato recorded at different growth stages excepting 70 DAP was more when the plants received two foliar spray of PS than without foliar spray (Table 1).

Regarding combination of different factors, application of $\mathrm{RD}_{\mathrm{NP}} \mathrm{K}_{\mathrm{MOP}} f b 2$ FS recorded significantly higher plant height at 30 DAP and it was $16 \%$ higher than that the conventional nutrient management $\left(\mathrm{RD}_{\mathrm{NP}} \mathrm{K}_{\mathrm{MOP}}\right)$ recorded (Table 2). But at later stages of crop growth (50, 70 and $90 \mathrm{DAP})$ application of $\mathrm{RD}_{\mathrm{NP}} \mathrm{K}_{\mathrm{PS}} f b 2 \mathrm{FS}$ recorded the maximum plant height among the different treatment combinations. As evident from Table 1, different sources and dose of $\mathrm{K}$ fertilization did not significantly influence the number of compound leaves plant ${ }^{-1}$ throughout the crop growth period, though two sprayings with PS exerted positive response on the number of compound leaf at active growth periods (50 and 70 DAP). 
Table 1 Direct effect of source, dose and method of potassium fertilization on germination, plant height, number of compound leaves plant ${ }^{-1}$ and leaf area index (LAI) of potato (cv. Kufri Jyoti). Values represent mean of pooled data of 2014-15 and 2015-16.

\begin{tabular}{|c|c|c|c|c|c|c|c|c|c|c|c|c|c|}
\hline \multirow[t]{3}{*}{ Fertilizer treatment } & Germination & \multicolumn{4}{|c|}{ Plant height (cm) } & \multicolumn{4}{|c|}{ Number of compound leaves plant ${ }^{-1}$} & \multicolumn{4}{|c|}{ LAI } \\
\hline & \multicolumn{13}{|c|}{ Days after planting } \\
\hline & 30 & 30 & 50 & 70 & 90 & 30 & 50 & 70 & 90 & 30 & 50 & 70 & 90 \\
\hline \multicolumn{14}{|l|}{ Source of $K$ fertilization } \\
\hline${ }^{\dagger} \mathrm{MOP}$ & 96.7 & 14.8 & $26.6^{\mathrm{b}}$ & $40.1^{\mathrm{b}}$ & $51.0^{\mathrm{b}}$ & 32.5 & 52.3 & 47.2 & 28.3 & 0.89 & $3.20^{\mathrm{b}}$ & $3.01^{\mathrm{b}}$ & $2.62^{b}$ \\
\hline${ }^{\dagger} \mathrm{PS}$ & 96.7 & 14.9 & $27.7^{\mathrm{a}}$ & $42.7^{\mathrm{a}}$ & $53.0^{\mathrm{a}}$ & 35.4 & 54.2 & 48.3 & 31.4 & 0.84 & $3.40^{\mathrm{a}}$ & $3.18^{\mathrm{a}}$ & $2.84^{\mathrm{a}}$ \\
\hline SEm \pm & 0.51 & 0.19 & 0.28 & 0.37 & 1.17 & 1.68 & 1.53 & 1.57 & 1.31 & 0.05 & 0.04 & 0.03 & 0.05 \\
\hline $\mathrm{CD}(p \leq 0.05)$ & $\mathrm{NS}$ & $\mathrm{NS}$ & 0.85 & 1.14 & 0.38 & $\mathrm{NS}$ & NS & NS & NS & $\mathrm{NS}$ & 0.12 & 0.10 & 0.15 \\
\hline \multicolumn{14}{|l|}{ Dose of $K$ fertilization } \\
\hline${ }^{*} \mathrm{RD}_{\mathrm{NPK}}$ & 97.3 & 15.0 & $28.0^{\mathrm{a}}$ & $42.8^{\mathrm{a}}$ & 52.1 & 33.4 & 54.3 & 49.2 & 31.8 & 0.88 & $3.36^{\mathrm{a}}$ & 3.13 & $2.81^{\mathrm{a}}$ \\
\hline $\mathrm{RD}_{\mathrm{NP}} 3 / 4 \mathrm{~K}$ & 96.1 & 14.8 & $26.3^{\mathrm{b}}$ & $40.1^{\mathrm{b}}$ & 51.9 & 34.5 & 52.1 & 46.3 & 27.9 & 0.85 & $3.24^{\mathrm{b}}$ & 3.06 & $2.65^{\mathrm{b}}$ \\
\hline SEm \pm & 0.52 & 0.19 & 0.28 & 0.38 & 0.38 & 1.68 & 1.53 & 1.57 & 1.31 & 0.05 & 0.04 & 0.03 & 0.05 \\
\hline $\mathrm{CD}(p \leq 0.05)$ & $\mathrm{NS}$ & NS & 0.85 & 1.14 & NS & $\mathrm{NS}$ & NS & NS & NS & NS & 0.12 & NS & 0.15 \\
\hline \multicolumn{14}{|l|}{ Foliar of $K$ fertilization } \\
\hline Without foliar spray & 96.9 & 14.5 & $25.1^{\mathrm{b}}$ & 40.9 & $50.1^{\mathrm{b}}$ & 34.4 & $45.9^{\mathrm{b}}$ & $42.3^{\mathrm{b}}$ & 30.3 & 0.84 & $3.09^{\mathrm{b}}$ & $2.94^{\mathrm{b}}$ & $2.50^{\mathrm{b}}$ \\
\hline${ }^{\S}$ Two spray with PS & 96.6 & 15.2 & $29.2^{\mathrm{a}}$ & 41.9 & $54.0^{\mathrm{a}}$ & 33.5 & $60.5^{\mathrm{a}}$ & $53.2^{\mathrm{a}}$ & 29.4 & 0.89 & $3.51^{\mathrm{a}}$ & $3.25^{\mathrm{a}}$ & $2.96^{\mathrm{a}}$ \\
\hline SEm \pm & 0.52 & 0.18 & 0.27 & 0.38 & 0.38 & 1.67 & 1.52 & 1.57 & 1.30 & 0.05 & 0.04 & 0.03 & 0.05 \\
\hline $\mathrm{CD}(p \leq 0.05)$ & NS & NS & 0.85 & NS & 1.18 & NS & 4.64 & 4.78 & NS & NS & 0.11 & NS & 0.15 \\
\hline
\end{tabular}

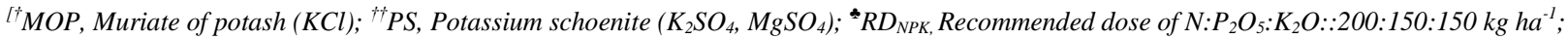
${ }^{\S}$ Two foliar spray of $1 \%$ PS at 30 and 50 days after planting; NS, Non-significant; Means followed by a different letter are significantly different at $p \leq 0.05$ by Tukey's HSD (honest significant difference) test].

Table 2 Interaction effect of source, dose and method of potassium fertilization on germination, plant height, number of compound leaves/plant and leaf area index (LAI) of potato (cv. Kufri Jyoti). Values represent mean of pooled data of 2014-15 and 2015-16.

\begin{tabular}{|c|c|c|c|c|c|c|c|c|c|c|c|c|c|}
\hline \multirow[t]{3}{*}{$\begin{array}{l}\text { Fertilizer } \\
\text { treatment }\end{array}$} & $\begin{array}{c}\text { Germination } \\
\%\end{array}$ & \multicolumn{4}{|c|}{ Plant height $(\mathrm{cm})$} & \multicolumn{4}{|c|}{$\begin{array}{l}\text { Number of compound leaves } \\
\text { plant }^{-1}\end{array}$} & \multicolumn{4}{|c|}{ LAI } \\
\hline & \multicolumn{13}{|c|}{ Days after planting } \\
\hline & 30 & 30 & 50 & 70 & 90 & 30 & 50 & 70 & 90 & 30 & 50 & 70 & 90 \\
\hline $\mathrm{RD}_{\mathrm{NP}} \mathrm{K}_{\mathrm{MOP}}$ & 97.6 & $13.7^{\mathrm{b}}$ & $25.1^{\mathrm{de}}$ & $41.7^{\mathrm{ab}}$ & $48.9^{\mathrm{b}}$ & 32.0 & $46.7^{\mathrm{bc}}$ & $44.3^{\mathrm{ab}}$ & 33.7 & 0.83 & $3.15^{\mathrm{b}}$ & $2.94^{\mathrm{c}}$ & $2.48^{\mathrm{c}}$ \\
\hline $\begin{array}{l}\mathrm{RD}_{\mathrm{NP}} \mathrm{K}_{\mathrm{MOP}} f b \\
2 \mathrm{FS}^{*}\end{array}$ & 97.0 & $15.9^{\mathrm{a}}$ & $29.8^{\mathrm{ab}}$ & $41.6^{\mathrm{ab}}$ & $53.1^{\mathrm{ab}}$ & 32.7 & $59.7^{\mathrm{ab}}$ & $52.0^{\mathrm{ab}}$ & 28.7 & 0.94 & $3.39^{\mathrm{ab}}$ & $3.23^{\mathrm{abc}}$ & $2.97^{\mathrm{ab}}$ \\
\hline $\mathrm{RD}_{\mathrm{NP}} 3 / 4 \mathrm{~K}_{\mathrm{MOP}}$ & 94.7 & $14.6^{\mathrm{ab}}$ & $22.8^{\mathrm{e}}$ & $37.6^{\mathrm{b}}$ & $49.2^{\mathrm{b}}$ & 32.3 & $43.3^{\mathrm{bc}}$ & $40.7^{\mathrm{b}}$ & 24.3 & 0.84 & $3.09^{\mathrm{b}}$ & $2.95^{\mathrm{c}}$ & $2.54^{\mathrm{bc}}$ \\
\hline $\begin{array}{l}\mathrm{RD}_{\mathrm{NP}} 3 / 4 \mathrm{~K}_{\mathrm{MOP}} \\
f b 2 \mathrm{FS}\end{array}$ & 97.7 & $15.0^{\mathrm{ab}}$ & $28.5^{\mathrm{abc}}$ & $39.7^{\mathrm{ab}}$ & $53.0^{\mathrm{ab}}$ & 33.0 & $59.3^{\mathrm{ab}}$ & $51.7^{\mathrm{ab}}$ & 26.7 & 0.96 & $3.16^{\mathrm{b}}$ & $2.93^{\mathrm{c}}$ & $2.49^{\mathrm{a}}$ \\
\hline $\mathrm{RD}_{\mathrm{NP}} \mathrm{K}_{\mathrm{PS}}$ & 98.3 & $14.6^{\mathrm{ab}}$ & $26.6^{\text {bcd }}$ & $43.0^{\mathrm{ab}}$ & $51.2^{\mathrm{ab}}$ & 34.3 & $48.0^{b c}$ & $44.7 \mathrm{a}^{\mathrm{b}}$ & 32.7 & 0.85 & $3.08^{\mathrm{b}}$ & $2.99^{b c}$ & $2.62^{\mathrm{bc}}$ \\
\hline $\begin{array}{l}\mathrm{RD}_{\mathrm{NP}} \mathrm{K}_{\mathrm{PS}} f b 2 \\
\mathrm{FS}\end{array}$ & 96.4 & $15.6^{\mathrm{ab}}$ & $30.4^{\mathrm{a}}$ & $44.9^{\mathrm{a}}$ & $55.2^{\mathrm{a}}$ & 34.7 & $63.0^{\mathrm{a}}$ & $55.7^{\mathrm{a}}$ & 32.3 & 0.91 & $3.82^{\mathrm{a}}$ & $3.37^{\mathrm{ab}}$ & $3.17^{\mathrm{a}}$ \\
\hline $\mathrm{RD}_{\mathrm{NP}} 3 / 4 \mathrm{~K}_{\mathrm{PS}}$ & 96.9 & $15.0^{\mathrm{ab}}$ & $25.8^{\text {cde }}$ & $41.4^{\mathrm{ab}}$ & $50.9^{\mathrm{ab}}$ & 39.0 & $45.7^{\mathrm{bc}}$ & $39.7^{\mathrm{b}}$ & 30.7 & 0.85 & $3.05^{\mathrm{b}}$ & $2.88^{\mathrm{c}}$ & $2.36^{\mathrm{c}}$ \\
\hline $\begin{array}{l}\mathrm{RD}_{\mathrm{NP}} 3 / 4 \mathrm{~K}_{\mathrm{PS}} f b \\
2 \mathrm{FS}\end{array}$ & 95.4 & $14.5^{\mathrm{ab}}$ & $28.1^{\mathrm{abcd}}$ & $41.6^{\mathrm{ab}}$ & $54.6^{\mathrm{a}}$ & 33.7 & $60.0^{\mathrm{ab}}$ & $53.3^{\mathrm{ab}}$ & 30.0 & 0.74 & $3.66^{\mathrm{a}}$ & $3.47^{\mathrm{a}}$ & $3.22^{\mathrm{a}}$ \\
\hline SEm \pm & 1.02 & 0.37 & 0.56 & 0.75 & 0.77 & 3.37 & 3.01 & 3.15 & 2.61 & 0.10 & 0.08 & 0.06 & 0.10 \\
\hline $\mathrm{CD}(p \leq 0.05)$ & NS & 1.90 & 3.32 & 5.13 & 5.11 & NS & 18.9 & 14.5 & NS & NS & 0.59 & 0.30 & 0.30 \\
\hline
\end{tabular}

[RD/recommended dose of fertilizer $\left(\mathrm{N}: \mathrm{P}_{2} \mathrm{O}_{5}\right), 200: 150 \mathrm{~kg} \mathrm{ha} a^{-1} ; \mathrm{NS}$, Non-significant; Means followed by a different letter are significantly different at $p \leq 0.05$ by Tukey's HSD (honest significant difference) test; * 2 FS, Two foliar spray of $1 \%$ PS at 30 and 50 days after planting $]$ 
Table 3 Direct effect of source, dose and method of potassium fertilization on haulm dry weight, tuber dry weight and total (haulm + tuber) dry weight of potato (cv. Kufri Jyoti). Values represent mean of pooled data of 2014-15 and 2015-16.

\begin{tabular}{|c|c|c|c|c|c|c|c|c|c|c|c|}
\hline \multirow{3}{*}{$\begin{array}{l}\text { Fertilizer } \\
\text { treatment }\end{array}$} & \multicolumn{4}{|c|}{ Haulm dry weight $\left(\mathrm{g} \mathrm{m}^{-2}\right)$} & \multicolumn{3}{|c|}{ Tuber dry weight $\left(\mathrm{g} \mathrm{m}^{-2}\right)$} & \multicolumn{4}{|c|}{ Total dry weight $\left(\mathrm{g} \mathrm{m}^{-2}\right)$} \\
\hline & \multicolumn{11}{|c|}{ Days after planting } \\
\hline & 30 & 50 & 70 & 90 & 50 & 70 & 90 & 30 & 50 & 70 & 90 \\
\hline \multicolumn{12}{|c|}{ Factor A: Source } \\
\hline${ }^{\dagger} \mathrm{MOP}$ & 9.04 & $118^{\mathrm{a}}$ & $187^{\mathrm{b}}$ & $164^{\mathrm{b}}$ & 534 & 969 & $2072^{\mathrm{b}}$ & 9.04 & $652^{\mathrm{a}}$ & 1156 & $2236^{\mathrm{b}}$ \\
\hline${ }^{\dagger \dagger} \mathrm{PS}$ & 8.96 & $92.4^{\mathrm{b}}$ & $199^{\mathrm{a}}$ & $174^{\mathrm{a}}$ & 538 & 978 & $2154^{\mathrm{a}}$ & 8.96 & $630^{\mathrm{b}}$ & 1177 & $2329^{\mathrm{a}}$ \\
\hline SEm \pm & 0.15 & 1.52 & 2.11 & 2.52 & 3.70 & 8.92 & 14.76 & 0.15 & 3.42 & 8.69 & 15.70 \\
\hline $\mathrm{CD}(p \leq 0.05)$ & NS & 4.61 & 6.40 & 7.63 & NS & NS & 44.75 & NS & 10.38 & NS & 47.61 \\
\hline \multicolumn{12}{|c|}{ Factor B: Dose } \\
\hline${ }^{2} \mathrm{RD}_{\mathrm{NPK}}$ & 9.08 & $121^{\mathrm{a}}$ & $199^{\mathrm{a}}$ & $174^{\mathrm{a}}$ & 540 & $1007^{\mathrm{a}}$ & $2165^{\mathrm{a}}$ & 9.08 & $661^{\mathrm{a}}$ & $1206^{\mathrm{a}}$ & $2339^{\mathrm{a}}$ \\
\hline $\mathrm{RD}_{\mathrm{NP}} 3 / 4 \mathrm{~K}$ & 9.91 & $88.7^{\mathrm{b}}$ & $187^{\mathrm{b}}$ & $164^{\mathrm{b}}$ & 532 & $940^{\mathrm{b}}$ & $2062^{\mathrm{b}}$ & 9.91 & $621^{\mathrm{b}}$ & $1127^{\mathrm{b}}$ & $2226^{\mathrm{b}}$ \\
\hline SEm \pm & 0.15 & 1.52 & 2.11 & 2.51 & 3.71 & 8.90 & 14.77 & 0.15 & 3.46 & 8.90 & 15.69 \\
\hline $\mathrm{CD}(p \leq 0.05)$ & $\mathrm{NS}$ & 4.60 & 6.40 & 7.61 & NS & 27.05 & 44.70 & $\mathrm{NS}$ & 10.38 & 26.38 & 47.60 \\
\hline \multicolumn{12}{|c|}{ Factor C: Method } \\
\hline Without & $8.36^{\mathrm{b}}$ & $115^{\mathrm{a}}$ & $182^{\mathrm{b}}$ & $159^{\mathrm{b}}$ & 533 & $939^{\mathrm{b}}$ & $2047^{\mathrm{b}}$ & $8.36^{\mathrm{b}}$ & $647^{\mathrm{a}}$ & $1120^{\mathrm{b}}$ & $2206^{\mathrm{b}}$ \\
\hline${ }^{\S}$ Two spray & $9.64^{\mathrm{a}}$ & $95.3^{\mathrm{b}}$ & $204^{\mathrm{a}}$ & $179^{\mathrm{a}}$ & 540 & $1008^{\mathrm{a}}$ & $2180^{\mathrm{a}}$ & $9.64^{\mathrm{a}}$ & $635^{\mathrm{b}}$ & $1213^{\mathrm{a}}$ & $2359^{\mathrm{a}}$ \\
\hline SEm \pm & 0.15 & 1.52 & 2.12 & 2.50 & 3.73 & 8.90 & 14.79 & 0.15 & 3.41 & 8.70 & 15.70 \\
\hline $\mathrm{CD}(p \leq 0.05)$ & 0.45 & 4.60 & 6.35 & 7.63 & NS & 27.06 & 44.40 & 0.45 & 10.30 & 26.39 & 47.63 \\
\hline
\end{tabular}

$l^{\dagger} \mathrm{MOP}$, Muriate of potash $(\mathrm{KCl}) ;{ }^{\dagger \dagger} \mathrm{PS}$, Potassium schoenite $\left(\mathrm{K}_{2} \mathrm{SO}_{4}, \mathrm{MgSO}_{4}\right) ;{ }^{\star} \mathrm{RD}_{\mathrm{NPK}}$, Recommended dose of $\mathrm{N}_{2} \mathrm{P}_{2} \mathrm{O}_{5}: \mathrm{K}_{2} \mathrm{O}:: 200: 150: 150 \mathrm{~kg}$ hal ;

${ }^{\S}$ Two foliar spray of $1 \%$ PS at 30 and 50 days after planting; NS, Non-significant; Means followed by a different letter are significantly different at $p \leq 0.05$ by Tukey's HSD (honest significant difference) test.]

Table 4 Interaction effect of source, dose and method of potassium fertilization on haulm dry weight, tuber dry weight and total (haulm + tuber) dry weight of potato (cv. Kufri Jyoti). Values represent mean of pooled data of 2014-15 and 2015-16.

\begin{tabular}{|c|c|c|c|c|c|c|c|c|c|c|c|}
\hline \multirow{3}{*}{$\begin{array}{l}\text { Fertilizer } \\
\text { treatment }\end{array}$} & \multicolumn{4}{|c|}{ Haulm dry weight $\left(\mathrm{g} \mathrm{m}^{-2}\right)$} & \multicolumn{3}{|c|}{ Tuber dry weight $\left(\mathrm{g} \mathrm{m}^{-2}\right)$} & \multicolumn{4}{|c|}{ Total dry weight $\left(\mathrm{g} \mathrm{m}^{-2}\right)$} \\
\hline & \multicolumn{11}{|c|}{ Days after planting } \\
\hline & 30 & 50 & 70 & 90 & 50 & 70 & 90 & 30 & 50 & 70 & 90 \\
\hline $\mathrm{RD}_{\mathrm{NP}} \mathrm{K}_{\mathrm{MOP}}$ & $8.37^{\mathrm{c}}$ & $118^{\mathrm{ab}}$ & $174^{\mathrm{d}}$ & $154^{\text {cd }}$ & 533 & $974^{\text {bcd }}$ & $2072^{\mathrm{b}}$ & $8.37^{\mathrm{c}}$ & $650^{\mathrm{bc}}$ & $1148^{\text {cd }}$ & $2226^{\mathrm{c}}$ \\
\hline $\mathrm{RD}_{\mathrm{NP}} \mathrm{K}_{\mathrm{MOP}} f b 2 \mathrm{FS} *$ & $9.14^{\mathrm{abc}}$ & $125^{\mathrm{a}}$ & $212^{\mathrm{ab}}$ & $182^{\mathrm{ab}}$ & 539 & $1007^{\mathrm{ab}}$ & $2164^{\mathrm{ab}}$ & $9.14^{\mathrm{abc}}$ & $664^{\mathrm{ab}}$ & $1219^{\mathrm{b}}$ & $2346^{\mathrm{b}}$ \\
\hline $\mathrm{RD}_{\mathrm{NP}} 3 / 4 \mathrm{~K}_{\mathrm{MOP}}$ & $8.23^{\mathrm{c}}$ & $106^{\mathrm{b}}$ & $172^{\mathrm{d}}$ & $148^{\mathrm{d}}$ & 532 & $904^{\mathrm{d}}$ & $1999^{\mathrm{b}}$ & $8.23^{\mathrm{c}}$ & $639^{c}$ & $1077^{\mathrm{e}}$ & $2147^{\mathrm{e}}$ \\
\hline $\mathrm{RD}_{\mathrm{NP}}{ }^{3 / 4 \mathrm{~K}_{\mathrm{MOP}}} f b 2 \mathrm{FS}$ & $10.40^{\mathrm{ab}}$ & $120^{\mathrm{ab}}$ & $189^{\text {cd }}$ & $171^{\text {bcd }}$ & 531 & $991^{\mathrm{bc}}$ & $2053^{\mathrm{b}}$ & $10.40^{\mathrm{ab}}$ & $652^{\mathrm{bc}}$ & $1180^{\mathrm{bc}}$ & $2225^{\mathrm{c}}$ \\
\hline $\mathrm{RD}_{\mathrm{NP}} \mathrm{K}_{\mathrm{PS}}$ & $8.23^{\mathrm{c}}$ & $115^{\mathrm{ab}}$ & $185^{\text {cd }}$ & $160^{\text {bcd }}$ & 531 & $964^{\text {bcd }}$ & $2081^{\mathrm{b}}$ & $8.23^{\mathrm{c}}$ & $646^{\mathrm{bc}}$ & $1149^{\text {cd }}$ & $2241^{\mathrm{c}}$ \\
\hline $\mathrm{RD}_{\mathrm{NP}} \mathrm{K}_{\mathrm{PS}} f b 2 \mathrm{FS}$ & $10.60^{\mathrm{a}}$ & $127^{\mathrm{a}}$ & $225^{\mathrm{a}}$ & $201^{\mathrm{a}}$ & 555 & $1082^{\mathrm{a}}$ & $2340^{\mathrm{a}}$ & $10.60^{\mathrm{a}}$ & $682^{\mathrm{a}}$ & $1307^{\mathrm{a}}$ & $2541^{\mathrm{a}}$ \\
\hline $\mathrm{RD}_{\mathrm{NP}} 3 / 4 \mathrm{~K}_{\mathrm{PS}}$ & $8.60^{\mathrm{abc}}$ & $119^{\mathrm{ab}}$ & $195^{\mathrm{bc}}$ & $174^{\text {bc }}$ & 534 & $911^{\text {cd }}$ & $2035^{\mathrm{b}}$ & $8.60 \mathrm{abc}$ & $652^{\mathrm{bc}}$ & $1106^{\mathrm{de}}$ & $2210^{\mathrm{d}}$ \\
\hline $\mathrm{RD}_{\mathrm{NP}}{ }^{3 / 4} \mathrm{~K}_{\mathrm{PS}} f b 2 \mathrm{FS}$ & $8.42^{\mathrm{bc}}$ & $123^{\mathrm{ab}}$ & $190^{\text {cd }}$ & $161^{\text {bcd }}$ & 532 & $952^{\text {bcd }}$ & $2160^{\mathrm{b}}$ & $8.42^{\mathrm{bc}}$ & $655^{\mathrm{bc}}$ & $1142^{\text {cd }}$ & $2322^{\mathrm{b}}$ \\
\hline $\mathrm{SEm} \pm$ & 0.29 & 3.04 & 4.22 & 5.03 & 7.40 & 17.84 & 29.51 & 0.29 & 6.85 & 17.40 & 31.40 \\
\hline $\mathrm{CD}(p \leq 0.05)$ & 0.89 & 9.31 & 18.2 & 25.3 & NS & 51.26 & 179.2 & 0.89 & 41.5 & 53.29 & 96.14 \\
\hline
\end{tabular}

[RDF/recommended dose of fertilizer $\left(\mathrm{N}: \mathrm{P}_{2} \mathrm{O}_{5}\right), 200: 150 \mathrm{~kg} \mathrm{ha}^{-1} ; \mathrm{NS}$, Non-significant; Means followed by a different letter are significantly different at $p \leq 0.05$ by Tukey's HSD (honest significant difference) test; * 2 FS, Two foliar spray of $1 \%$ PS at 30 and 50 days after planting.]

In harmony to these findings, results obtained from different treatment combinations revealed that, potato fertilized with $\mathrm{RD}_{\mathrm{NP}} \mathrm{K}_{\mathrm{PS}} f b 2$ FS produced significantly $(p \leq 0.05)$ higher number of compound leaves plant ${ }^{-1}$ at 50 and 70 DAP and these were 35 and $26 \%$ higher than those in potato fertilized with conventional nutrient management (Table 2). At the stage of maturity (90 DAP), senescence resulted in lowering in the number of compound leaves naturally, reflecting the non significance of all the fertilization treatments. LAI increased progressively over the time upto 50 DAP irrespective of source, dose and foliar application of $\mathrm{K}$ fertilizers, and then it declined gradually (Table 1). Plants fertilized with PS exerted positive response on LAI throughout the growth period except at early growth stage (30 DAP). Reduction in the $\mathrm{K}$ fertilizer dose $\left(\mathrm{RD}_{\mathrm{NP}} \mathrm{s}_{\mathrm{K}}\right)$ recorded significantly lower LAI value at 50 and 90 DAP over recommended dose. The addition of PS through foliar application increased the growth of potato leaf up to 90 DAP as compared to without foliar PS application. As depicted from Table 2, different treatment combinations has no impact on LAI at the earlier crop growth stage; whereas, at later stages the maximum LAI was observed with $\mathrm{RD}_{\mathrm{NP}} \mathrm{K}_{\mathrm{PS}} f b 2 \mathrm{FS}$. 
Table 5 Direct effect of source, dose and method of potassium fertilization on crop growth rate (CGR), tuber bulking rate (TBR), tuber number and tuber yield of potato (cv. Kufri Jyoti). Values represent mean of pooled data of 2014-15 and 2015-16.

\begin{tabular}{|c|c|c|c|c|c|c|c|}
\hline \multirow[t]{3}{*}{ Fertilizer treatment } & \multicolumn{3}{|c|}{ Crop growth rate $\left(\mathrm{g} \mathrm{m}^{-2} \mathrm{day}^{-1}\right)$} & \multicolumn{2}{|c|}{ Tuber bulking rate $\left(\mathrm{g} \mathrm{m}^{-2}\right.$ day $\left.^{-1}\right)$} & \multirow{3}{*}{$\begin{array}{c}\text { Tuber } \\
\text { number ha }{ }^{-1} \\
\left(\times 10^{5}\right)\end{array}$} & \multirow{3}{*}{$\begin{array}{c}\text { Tuber yield ( } t \\
\left.\mathbf{h a}^{-1}\right)\end{array}$} \\
\hline & \multicolumn{5}{|c|}{ Days after planting } & & \\
\hline & $30-50$ & $50-70$ & $70-90$ & $50-70$ & $70-90$ & & \\
\hline \multicolumn{8}{|l|}{ Factor A: Source } \\
\hline${ }^{\dagger} \mathrm{MOP}$ & 5.43 & 3.47 & -1.16 & $86.1^{\mathrm{b}}$ & $26.4^{\mathrm{b}}$ & $4.42^{\mathrm{b}}$ & $22.4^{\mathrm{b}}$ \\
\hline †PS & 5.61 & 3.90 & -1.23 & $88.8^{\mathrm{a}}$ & $27.4^{\mathrm{a}}$ & $4.58^{\mathrm{a}}$ & $23.0^{\mathrm{a}}$ \\
\hline $\mathrm{SEm} \pm$ & 0.09 & 0.15 & 0.07 & 0.84 & 0.26 & 0.02 & 0.13 \\
\hline $\mathrm{CD}(p \leq 0.05)$ & NS & NS & NS & 2.57 & 0.78 & 0.07 & 0.39 \\
\hline \multicolumn{8}{|l|}{ Factor B: Dose } \\
\hline${ }^{2} \mathrm{RD}_{\mathrm{NPK}}$ & 5.61 & 3.89 & -1.24 & 88.3 & $27.3^{\mathrm{a}}$ & $4.55^{\mathrm{a}}$ & $23.5^{\mathrm{a}}$ \\
\hline $\mathrm{RD}_{\mathrm{NP}} 3 / 4 \mathrm{~K}$ & 5.42 & 3.84 & -1.46 & 86.6 & $26.5^{\mathrm{b}}$ & $4.45^{\mathrm{b}}$ & $21.9^{\mathrm{b}}$ \\
\hline SEm \pm & 0.08 & 0.15 & 0.07 & 0.83 & 0.25 & 0.02 & 0.13 \\
\hline $\mathrm{CD}(p \leq 0.05)$ & $\mathrm{NS}$ & NS & $\mathrm{NS}$ & $\mathrm{NS}$ & 0.79 & 0.06 & 0.39 \\
\hline \multicolumn{8}{|l|}{ Factor C: Method } \\
\hline Without foliar spray & $5.32^{\mathrm{b}}$ & $3.35^{\mathrm{b}}$ & -1.12 & 86.3 & $25.7^{\mathrm{b}}$ & $4.19^{b}$ & $21.9^{\mathrm{b}}$ \\
\hline${ }^{\S}$ Two spray with PS & $5.71^{\mathrm{a}}$ & $4.02^{\mathrm{a}}$ & -1.27 & 88.6 & $28.0^{\mathrm{a}}$ & $4.81^{\mathrm{a}}$ & $23.5^{\mathrm{a}}$ \\
\hline SEm \pm & 0.09 & 0.16 & 0.06 & 0.83 & 0.25 & 0.02 & 0.14 \\
\hline $\mathrm{CD}(p \leq 0.05)$ & 0.26 & 0.46 & NS & NS & 0.78 & 0.07 & 0.40 \\
\hline
\end{tabular}

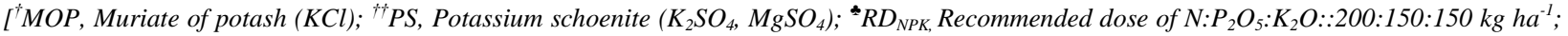

${ }^{s}$ Two foliar spray of $1 \%$ PS at 30 and 50 days after planting; NS, Non-significant; Means followed by a different letter are significantly different at $p \leq 0.05$ by Tukey's HSD (honest significant difference) test.]

Table 6 Interaction effect of source, dose and method of potassium fertilization on crop growth rate (CGR), tuber bulking rate (TBR), tuber number and tuber yield of potato (cv. Kufri Jyoti). Values represent mean of pooled data of 2014-15 and 2015-16.

\begin{tabular}{|c|c|c|c|c|c|c|c|}
\hline \multirow[t]{3}{*}{ Fertilizer treatment } & \multicolumn{3}{|c|}{ Crop growth rate $\left(\mathrm{g} \mathrm{m}^{-2}\right.$ day $\left.^{-1}\right)$} & \multicolumn{2}{|c|}{ Tuber bulking rate $\left(\mathrm{g} \mathrm{m}^{-2}\right.$ day $\left.^{-1}\right)$} & \multirow{3}{*}{$\begin{array}{c}\text { Tuber } \\
\text { number ha } \\
\left(\times 10^{5}\right)\end{array}$} & \multirow[t]{3}{*}{ Tuber yield $\left(\mathrm{t} \mathrm{ha}^{-1}\right)$} \\
\hline & \multicolumn{5}{|c|}{ Days after planting } & & \\
\hline & $30-50$ & $50-70$ & $70-90$ & $50-70$ & $70-90$ & & \\
\hline $\mathbf{R D}_{\mathrm{NP}} \mathbf{K}_{\mathrm{MOP}}$ & $5.48^{\mathrm{ab}}$ & $2.80^{\mathrm{c}}$ & -1.00 & $86.6 \mathrm{a}$ & $25.3^{\mathrm{c}}$ & $4.18^{\mathrm{cd}}$ & $22.0^{\mathrm{bcd}}$ \\
\hline $\mathrm{RD}_{\mathrm{NP}} \mathrm{K}_{\mathrm{MOP}} f b 2 \mathrm{FS} *$ & $5.79^{\mathrm{a}}$ & $4.35^{\mathrm{ab}}$ & -1.50 & 87.6 & $27.7^{\mathrm{b}}$ & $4.67^{\mathrm{ab}}$ & $24.2^{\mathrm{a}}$ \\
\hline $\mathrm{RD}_{\mathrm{NP}} 3 / 4 \mathrm{~K}_{\mathrm{MOP}}$ & $4.92^{\mathrm{b}}$ & $3.28^{\mathrm{bc}}$ & -1.22 & 85.3 & $26.1^{\mathrm{bc}}$ & $4.00^{\mathrm{d}}$ & $21.3^{\mathrm{cd}}$ \\
\hline $\mathrm{RD}_{\mathrm{NP}} 3 / 4 \mathrm{~K}_{\mathrm{MOP}} f b 2 \mathrm{FS}$ & $5.51^{\mathrm{ab}}$ & $3.45^{\mathrm{bc}}$ & -0.92 & 84.9 & $26.3^{\mathrm{bc}}$ & $4.83^{\mathrm{a}}$ & $22.1^{\mathrm{bcd}}$ \\
\hline $\mathbf{R D}_{\mathrm{NP}} \mathrm{K}_{\mathrm{PS}}$ & $5.36^{\mathrm{ab}}$ & $3.48^{\mathrm{bc}}$ & -1.23 & 86.0 & $25.4^{\mathrm{bc}}$ & $4.45^{\mathrm{bc}}$ & $22.8^{\mathrm{b}}$ \\
\hline $\mathrm{RD}_{\mathrm{NP}} \mathrm{K}_{\mathrm{PS}} f b 2 \mathrm{FS}$ & $5.82^{\mathrm{a}}$ & $4.93^{\mathrm{a}}$ & -1.23 & 92.9 & $30.7^{\mathrm{a}}$ & $4.90^{\mathrm{a}}$ & $25.0^{\mathrm{a}}$ \\
\hline $\mathrm{RD}_{\mathrm{NP}}{ }^{3 / 4 K_{\mathrm{PS}}}$ & $5.52^{\mathrm{ab}}$ & $3.82^{\mathrm{abc}}$ & -1.03 & 87.4 & $26.2^{\mathrm{bc}}$ & $4.12^{\mathrm{cd}}$ & $21.5^{\mathrm{d}}$ \\
\hline $\mathrm{RD}_{\mathrm{NP}}{ }^{3 / 4 \mathrm{~K}_{\mathrm{PS}} f b} 2 \mathrm{FS}$ & $5.73^{\mathrm{ab}}$ & $3.35^{\mathrm{bc}}$ & -1.42 & 88.9 & $27.3^{\mathrm{bc}}$ & $4.83^{\mathrm{a}}$ & $22.6^{\mathrm{bc}}$ \\
\hline SEm \pm & 0.17 & 0.30 & 0.14 & 1.70 & 0.51 & 0.05 & 0.26 \\
\hline $\mathrm{CD}(p \leq 0.05)$ & 0.53 & 0.93 & NS & NS & 1.58 & 0.14 & 0.79 \\
\hline
\end{tabular}

[RDF/recommended dose of fertilizer $\left(\mathrm{N}_{\mathrm{P}} \mathrm{P}_{2} \mathrm{O}_{5}\right), 200: 150 \mathrm{~kg} \mathrm{ha}^{-1} ; \mathrm{NS}$, Non-significant; Means followed by a different letter are significantly different at $p \leq 0.05$ by Tukey's HSD (honest significant difference) test; * 2 FS, Two foliar spray of $1 \%$ PS at 30 and 50 days after planting.]

Haulm dry weight (above ground biomass) was increased upto 70 DAP and after that it was declined (Table 3). Moreover, different source, dose and foliar $\mathrm{K}$ fertilization exerted significant effect $(p \leq 0.05)$ on haulm dry matter production. As compared to the crop fertilized with MOP or reduced dose of K fertilizer or even with no foliar application, the crop receiving PS or $\mathrm{RD}_{\mathrm{NPK}}$ or foliar PS spray exhibited positive response regarding haulm dry weight at later growth stages. The crop fertilized with $\mathrm{RD}_{\mathrm{NPK}} f b 2 \mathrm{FS}$ recorded higher haulm dry weight throughout the crop growth period (Table 4). 
Table 7 Direct effects of source, dose and method of potassium fertilization on tuber quality attributes of potato (cv. Kufri Jyoti). Values represent mean of pooled data of 2014-15 and 2015-16.

\begin{tabular}{|c|c|c|c|}
\hline Fertilizer treatment & Ascorbic acid (mg $100 \mathrm{~g}^{-1}$ of fresh weight of tuber) & Specific weight $\left(\mathrm{g} \mathrm{cm}^{-3}\right)$ & Reducing sugar content (\%) \\
\hline \multicolumn{4}{|l|}{ Factor A: Source } \\
\hline${ }^{\dagger} \mathrm{MOP}$ & $13.5^{\mathrm{b}}$ & 1.063 & $0.241^{\mathrm{a}}$ \\
\hline${ }^{\dagger \dagger} \mathrm{PS}$ & $13.8^{\mathrm{a}}$ & 1.059 & $0.230^{\mathrm{b}}$ \\
\hline SEm \pm & 0.05 & 0.002 & 0.002 \\
\hline $\mathrm{CD}(p \leq 0.05)$ & 0.14 & NS & 0.007 \\
\hline \multicolumn{4}{|l|}{ Factor B: Dose } \\
\hline${ }^{*} \mathrm{RD}_{\mathrm{NPK}}$ & $13.7^{\mathrm{a}}$ & 1.061 & $0.230^{\mathrm{b}}$ \\
\hline $\mathrm{RD}_{\mathrm{NP}} 3 / 4 \mathrm{~K}$ & $13.5^{\mathrm{b}}$ & 1.061 & $0.241^{\mathrm{a}}$ \\
\hline SEm \pm & 0.05 & 0.002 & 0.003 \\
\hline $\mathrm{CD}(p \leq 0.05)$ & 0.15 & $\mathrm{NS}$ & 0.007 \\
\hline \multicolumn{4}{|l|}{ Factor C: Method } \\
\hline Without foliar spray & $13.1^{\mathrm{b}}$ & $1.045^{\mathrm{b}}$ & 0.236 \\
\hline${ }^{\S}$ Two spray with PS & $14.1^{\mathrm{a}}$ & $1.077^{\mathrm{a}}$ & 0.235 \\
\hline $\mathrm{SEm} \pm$ & 0.05 & 0.002 & 0.003 \\
\hline $\mathrm{CD}(p \leq 0.05)$ & 0.14 & 0.005 & NS \\
\hline
\end{tabular}

$\left[{ }^{\dagger} \mathrm{MOP}\right.$, Muriate of potash $(\mathrm{KCl}) ;{ }^{\dagger t} \mathrm{PS}$, Potassium schoenite $\left(\mathrm{K}_{2} \mathrm{SO}_{4}, \mathrm{MgSO}_{4}\right) ;{ }^{*} \mathrm{RD}_{\mathrm{NPK}}$ Recommended dose of $\mathrm{N}: \mathrm{P}_{2} \mathrm{O}_{5}: \mathrm{K}_{2} \mathrm{O}:: 200: 150: 150 \mathrm{~kg}$ hal ;

${ }^{s}$ Two foliar spray of $1 \%$ PS at 30 and 50 days after planting; NS, Non-significant; Means followed by a different letter are significantly different at $p \leq 0.05$ by Tukey's HSD (honest significant difference) test.]

Table 8 Interaction effect of source, dose and method of potassium fertilization on tuber quality attributes of potato (cv. Kufri Jyoti). Values represent mean of pooled data of 2014-15 and 2015-16.

\begin{tabular}{|c|c|c|c|}
\hline Fertilizer treatment & $\begin{array}{l}\text { Ascorbic acid ( } \mathrm{mg} 100 \mathrm{~g}^{-1} \text { of fresh } \\
\text { weight of tuber) }\end{array}$ & Specific weight $\left(\mathrm{g} \mathrm{cm}^{-3}\right)$ & $\begin{array}{c}\text { Reducing sugar content } \\
(\%)\end{array}$ \\
\hline $\mathrm{RD}_{\mathrm{NP}} \mathrm{K}_{\mathrm{MOP}}$ & $13.32^{\mathrm{ab}}$ & $1.051^{\mathrm{ab}}$ & $0.249^{\mathrm{a}}$ \\
\hline $\mathrm{RD}_{\mathrm{NP}} \mathrm{K}_{\mathrm{MOP}} f b 2 \mathrm{FS} *$ & $13.80^{\mathrm{ab}}$ & $1.071^{\mathrm{ab}}$ & $0.229^{\mathrm{ab}}$ \\
\hline $\mathrm{RD}_{\mathrm{NP}} 3 / 4 \mathrm{~K}_{\mathrm{MOP}}$ & $12.81^{\mathrm{b}}$ & $1.034^{\mathrm{b}}$ & $0.237^{\mathrm{ab}}$ \\
\hline $\mathrm{RD}_{\mathrm{NP}} 3 / 4 \mathrm{~K}_{\mathrm{MOP}} f b 2 \mathrm{FS}$ & $13.95^{\mathrm{ab}}$ & $1.086^{\mathrm{ab}}$ & $0.244^{\mathrm{ab}}$ \\
\hline $\mathrm{RD}_{\mathrm{NP}} \mathrm{K}_{\mathrm{PS}}$ & $13.18^{\mathrm{ab}}$ & $1.034^{\mathrm{b}}$ & $0.221^{\mathrm{b}}$ \\
\hline $\mathrm{RD}_{\mathrm{NP}} \mathrm{K}_{\mathrm{PS}} f b 2 \mathrm{FS}$ & $14.57^{\mathrm{a}}$ & $1.088^{\mathrm{a}}$ & $0.221^{\mathrm{b}}$ \\
\hline $\mathrm{RD}_{\mathrm{NP}} 3 / 4 \mathrm{~K}_{\mathrm{PS}}$ & $13.16^{\mathrm{ab}}$ & $1.061^{\mathrm{ab}}$ & $0.231^{\mathrm{ab}}$ \\
\hline $\mathrm{RD}_{\mathrm{NP}} 3 / 4 \mathrm{~K}_{\mathrm{PS}} f b 2 \mathrm{FS}$ & $14.25^{\mathrm{ab}}$ & $1.070^{\mathrm{ab}}$ & $0.245^{\mathrm{a}}$ \\
\hline $\mathrm{SEm} \pm$ & 0.09 & 0.003 & 0.005 \\
\hline $\mathrm{CD}(p \leq 0.05)$ & 1.58 & 0.053 & 0.020 \\
\hline
\end{tabular}

[RDF/recommended dose of fertilizer $\left(\mathrm{N}_{\mathrm{P}} \mathrm{P}_{2} \mathrm{O}_{5}\right), 200: 150 \mathrm{~kg} \mathrm{ha}$; $\mathrm{NS}$, Non-significant; Means followed by a different letter are significantly different at $p \leq 0.05$ by Tukey's HSD (honest significant difference) test; * 2 FS, Two foliar spray of $1 \%$ PS at 30 and 50 days after planting.]

Tuber dry weight also increased with the crop growth (Table 3), but at the later part of crop growth (i. e. 70 DAP) the rate of increment was more due to higher TBR. Source of K exhibited non-significant impact on tuber dry weight at earlier growth stages, whereas, at maturity (90 DAP) more tuber dry weight was obtained from the plots receiving PS. Dose and foliar application of $\mathrm{K}$ had no effect on tuber dry weight at initial crop growth period (30 DAP), but a significant effect was observed at later growth stages (Table 4). Regarding treatment combinations, tuber dry weight did not differ significantly at earlier crop growth stage (30 DAP); on the contrary, the plants receiving $\mathrm{RD}_{\mathrm{NPK}} f b 2 \mathrm{FS}$ recorded higher tuber dry weight at 70 and $90 \mathrm{DAP}$. As haulm and tuber dry matter were influenced significantly with source, dose and foliar $\mathrm{K}$ fertilization, naturally total dry matter (haulm + tuber) accumulation was also influenced significantly (Table 3). The maximum total dry weight was recorded with $\mathrm{RD}_{\mathrm{NP}} \mathrm{K}_{\mathrm{PS}} f b 2 \mathrm{FS}$ throughout the crop growth period.

As evident from Table 5, different source and dose of $\mathrm{K}$ revealed non-significant response to CGR throughout the crop growth period, whereas, foliar application of PS significantly improved the CGR at earlier or active crop growth stages, though at the later part it was reflected to be non significant. Though the variation in CGR amongst varied treatment combinations did not follow any specific trend the highest CGR was registered from the plant receiving $\mathrm{RD}_{\mathrm{NP}} K_{\mathrm{PS}} f b$ 2FS (Table 6). And at the maturity stage the treatment

Journal of Experimental Biology and Agricultural Sciences http://www.jebas.org 
combinations reflected non significance over CGR showing negative values irrespective of the treatment combinations. Regarding the source of K, PS influenced TBR throughout growth period; the dose of $\mathrm{K}$ as well as foliar spray of it significantly influenced the TBR at later growth stage of potato (Table 5). Up to 70 DAP, treatment combinations revealed non-significant $(p \leq$ 0.05 ) response for TBR of potato, whereas, at later growth stage application of $\mathrm{RD}_{\mathrm{NP}} \mathrm{K}_{\mathrm{PS}} f b 2 \mathrm{FS}$ resulted in significantly higher TBR as compared to the rest of the combinations (Table 6).

\subsection{Tuber number and yield}

Source, dose and foliar feeding of potassium had positive impact on tuber number as well as yield of potato (Table 5). Reduction of $25 \% \mathrm{~K}$ irrespective of source along with two foliar sprays through PS not only produced significantly higher tuber number as compared to $\mathrm{RD}_{\mathrm{NPK}}$ or $\mathrm{RD}_{\mathrm{NP}} 3 / 4 \mathrm{~K}$ (irrespective of source) but also performed statistically at par with $\mathrm{RD}_{\mathrm{NPK}} f b 2$ FS (Table 6).Unlike the effect of different treatment combinations on tuber number $\mathrm{ha}^{-1}$, the tuber yield received under the treatment combinations $\mathrm{RD}_{\mathrm{NPK}}$ (irrespective of $\mathrm{K}$ source) along with two foliar sprays of PS was recorded significantly higher than those received under the rest treatment combinations.

\subsection{Quality parameters of potato tubers}

Different post-harvest quality parameters of potato altered significantly by source, dose and foliar feeding of $\mathrm{K}$ (Table 7). Ascorbic acid content of potato tuber increased significantly using $\mathrm{PS}$ as K source, while reduction of $\mathrm{K}$ fertilization dose $\left(\mathrm{RD}_{\mathrm{NP}} \mathrm{p}^{3 / 4 \mathrm{~K}}\right)$ reduced Ascorbic acid content significantly. The ascorbic acid content was more (14.57 mg $100 \mathrm{~g}^{-1}$ of fresh weight) in potato tubers harvested from plants treated with $\mathrm{RD}_{\mathrm{NP}} \mathrm{K}_{\mathrm{PS}} f b$ 2FS (13.74\% more than $\mathrm{RD}_{\mathrm{NP}} 3 / 4 \mathrm{~K}_{\mathrm{MOP}}$ ) and it was statistically at par with rest other $\mathrm{K}$ fertilization treatments (Table 8). Source and dose of $\mathrm{K}$ fertilization revealed non-significant impact on specific weight of potato but foliar PS fertilization significantly improved specific weight than without $\mathrm{K}$ fertilization (Table 7). The specific gravity was more in potato tubers harvested from plants treated with $\mathrm{RD}_{\mathrm{NP}} \mathrm{K}_{\mathrm{PS}} f b$ 2FS (Table 8 ). The reduction in specific gravity was observed with reduced $\mathrm{K}$ fertilization dose and $\mathrm{RD}_{\mathrm{NP}} \mathrm{K}_{\mathrm{PS}}$. Source and dose of $\mathrm{K}$ fertilization significantly influenced reducing sugar content of potato but foliar spray of PS failed to record any change in reducing sugar content of tuber (Table 7). The lowest reducing sugar content $(0.221 \%$ of dry weight $)$ was recorded in potatoes harvested from the plants fertilized with $\mathrm{RD}_{\mathrm{NP}} \mathrm{K}_{\mathrm{PS}} f b$ 2FS (Table 8).

\section{Discussions}

Different source, dose as well as combination of different factors of $\mathrm{K}$ fertilization failed to record any significant changes in the final plant stand of potato (Table 1 and Table 2). These finding reflects that $\mathrm{K}$ has meagre or no-impact on plant germination. Researchers advocated that even omission of NPK did not exert any significant response to the germination of potato seed tuber (Kumar et al., 2008; Banerjee et al., 2016a). In another experiment Kumar et al. (2007) also suggested that different sources (chloride and sulphate) as well as time of application of $\mathrm{K}$ fertilizer have no significant role on plant emergence.

Source and dose of $\mathrm{K}$ fertilization significantly increased plant height of potato at later growth stages viz. 50, 70 and 90 DAP (Table 1). Amongst various fertilizer combinations, $\mathrm{RD}_{\mathrm{NP}} \mathrm{K}_{\mathrm{MOP}} f b 2$ FS increased the plant height by $16 \%$ over the conventional nutrient management practice i.e. $\mathrm{RD}_{\mathrm{NP}} \mathrm{K}_{\mathrm{MOP}}$ (Table 2). Apart from its $\mathrm{K}_{2} \mathrm{O}$ content, presence of $11 \% \mathrm{Mg}$ in PS plays an important role in vegetative growth of potato by increasing chlorophyll content in leaves and accumulating more photosynthates in plant tissue. This may be one of the major reasons behind such increment of plant height at later stages of growth of this tuber yielding plant (Sharma et al, 1998; Kumar et al., 2007).

As a matter of fact the number of compound leaves plant ${ }^{-1}$ was not significantly influenced by different sources and dose of $\mathrm{K}$ fertilization; while foliar application of PS exerted positive response on the number of compound leaf at active growth periods i.e. 50 and 70 DAP by 31.8 and $25.8 \%$ respectively over without foliar spraying. Different treatment combinations has a little impact on LAI at the earlier crop growth stage, whereas, at later stages the maximum LAI was observed with $\mathrm{RD}_{\mathrm{NP}} \mathrm{K}_{\mathrm{PS}} f b 2$ FS (Table 2). Similar results were observed by Rathore et al. (2014) who reported the non-significance of application of potassium schoenite on the number of leaves of groundnut. The findings like nonsignificance of different source and time of $\mathrm{K}$ fertilizer application on number of stem and compound leaf of potato cv. Kufri Chipsona-2 observed by Kumar et al. (2007) also corroborate these facts.

It is evident from Table 3 that schoenite fertilization either basal or foliar produced higher haulm, tuber as well as total dry matter accumulation. Plants receiving $\mathrm{RD}_{\mathrm{NP}} \mathrm{K}_{\mathrm{PS}} f b 2 \mathrm{FS}$ recorded the maximum tuber dry weight at later growth stages (70 and 90 DAP) by 11 and $13 \%$ respectively over conventionally grown potato tubers receiving $\mathrm{K}$ from chloride bearing $\mathrm{K}$-fertilizer. The higher uptake of chloride ion rather than $\mathrm{K}$ having negative impact on dry matter content of tuber may be the partial explanation behind such lower tuber dry-matter percentage associated with K-chloride fertilization (Toolangi, 1995). Unlike this potassic fertilizer reflecting a negative role of chloride as explained, the presence of sulphur in PS apart from $\mathrm{K}$ has a dual role in altering the metabolic activities of potato (Kumar et al., 2007).

The hydrolytic activity of carbohydrases like invertase, amylase and beta glucosidase are inhibited by chloride ions, whereas, increased by sulphate ions. In addition, sulphur has no negative role like chloride in the uptake of $\mathrm{K}$ (Smith, 1987). Thus, as a whole PS being a source of $\mathrm{K}$ and sulphur enhances the assimilation of metabolites and judicious translocation of phtosynthates from source to sink and ultimately enhanced the dry matter accumulation. Although at later growth stages different fertilizer combinations recorded non-significant response to the CGR (Table 5), the application of $\mathrm{RD}_{\mathrm{NP}} \mathrm{K}_{\mathrm{PS}} f b$ 2FS resulted in $21.6 \%$ higher TBR over the $\mathrm{RD}_{\mathrm{NP}} \mathrm{K}_{\mathrm{MOP}}$ did at $70-90$ DAP (Table $6)$. 
Source, dose and foliar fertilization of potassium recorded positive impact on both the number of tuber and yield of potato (Table 5). As matter of importance, two foliar applications of schoenite along with $25 \%$ reduced dose of $\mathrm{K}$ fertilizer produced significantly higher number of tuber as compared to $\mathrm{RD}_{\mathrm{NPK}}$ or $\mathrm{RD}_{\mathrm{NP}} 3 / 4 \mathrm{~K}$. On other hand application of $\mathrm{RD}_{\mathrm{NP}} \mathrm{K}_{\mathrm{PS}} f b 2 \mathrm{FS}$ resulted highest tuber production $(13.34 \%$ higher) than conventionally grown potato $\left(\mathrm{RD}_{\mathrm{NP}} \mathrm{K}_{\mathrm{MOP}}\right)$ (Table 6).

Moreover, for higher production foliar sprays with PS in combination with recommended basal doses were superior to omission of foliar spray. It may be due to the continuous supply of the essential plant nutrients like $\mathrm{K}, \mathrm{S}$ and $\mathrm{Mg}$ for a longer period of crop growth which possibly facilitates a steady translocation of the photosynthates to the underground parts resulting in an increase in tuber number and yield (Sarkar et al., 2007). In harmony to such findings, the observations of Rathore et al. (2014) may be cited. They recorded similar results in groundnut with potassium schoenite fertilization. The increment of tuber yield with sulphate form of $\mathrm{K}$ fertilizer i.e. PS may be attributed to the production of larger sized tubers having higher dry matter content than that produced by the crop fertilized with chloride from of $\mathrm{K}$ fertilizers i.e. MOP (Khan et al., 2012)

The processing quality of potato is mainly influenced by quality characters like specific gravity, dry matter and reducing sugar content in tubers (Abbas et al., 2011). Therefore, improvement of these quality parameters is the primary concern for potato processing industries. It is evident from the present study that quality of potato was improved with using PS as K source (Table 7). Ascorbic acid (vitamin C) is an essential vitamin in potato tubers (Hamouz et al., 2009) and $\mathrm{K}$ fertilization with PS significantly improves its content in tubers (Table 7). Application of increased amount of chloride of potash decreased the ascorbic acid concentration in potato tubers (Manolov et al., 2015). Specific weight is one of the most important factors to the potato processors, as the recovery of the finished product is directly linked to it. The maximum specific weight of potato tubers was recorded from the plots receiving $\mathrm{RD}_{\mathrm{NP}} \mathrm{K}_{\mathrm{PS}} f b 2 \mathrm{FS}$, while reduction in specific weight was observed with reduced doses of $\mathrm{K}$ fertilizer (Table 8). As compared to chloride form, sulphate form of Kfertilizer has positive impact on tuber specific weight (Kumar et al., 2007) through higher accumulation of dry matter.

Reducing sugar content in potato tubers greatly influences processing quality parameters of potato by regulating the colour of chips as well as nutritive and processing quality (Nikolova \& Blagoeva, 2000). Reducing sugars are critical precursors for acrylamide formation during frying which is considered carcinogenic and neurotoxic for humans (Zorb et al., 2014). Free reducing sugars also participate in Maillard reaction; thus, contribute to develop unfavourable colour and flavour. In present study, source and dose of $\mathrm{K}$ fertilization helps in decreasing reducing sugar content of potato tuber (Table 7). Kumar et al. (2007) also suggested that K-sulphate fertilization can be effectively adopted instead of K-chloride, as it increases tuber drymatter percentage and chips recovery and decreases chips oil percentage without yield compensation.

\section{Conclusions}

Thus from the results cited in detail and the facts discussed analytically it may be concluded that PS, being a source of good amount of potassium, magnesium and sulphur, helps in balanced assimilation, translocation and finally accumulation of photosynthates in potato. Recommended dose of NPK along with two foliar spray of PS improves the plant growth, yield and quality of potato tubers. So, farmers can use this fertilizer successfully in potato and PS may be an alternative choice as a source of potassium.

\section{Conflict of interest}

Authors would hereby like to declare that there is no conflict of interests that could possibly arise.

\section{References}

Abbas G, Farooq K, Hafiz IA, Hussain A, Abbasi NA, Shabbir G (2011) Assessment of processing and nutritional quality of potato genotypes in Pakistan. Pakistan Journal of Agricultural Science 48: 169-175.

Alva A, Fan M, Qing C, Rosen C, Ren H (2011) Improving nutrient use efficiency in Chinese potato production: Experiences from the United States. Journal of Crop Improvement 25: 46-85. DOI: $10.1080 / 15427528.2011 .538465$

Banerjee H, Dutta S, Rana L, Ray K, Sarkar S. Majumder M (2016b) Fertilization Impacts on Productivity and Profitability of Potato. Better Crops with Plant Food 100: 7-9.

Banerjee H, Rana L, Ray K, Sarkar S, Bhattacharyya K, Dutta SK (2016a) Differential physiological response in potato (Solanum tuberosum L.) upon exposure to nutrient omissions. Indian Journal of Plant Physiology 21: 129-136. DOI: 10.1007/s40502-016-0211-x

Casanas R, González M, Rodríguez E, Marrero A, Díaz C (2002) Chemometric studies of chemical compounds in five cultivars of potatoes from Tenerife. Journal of Agricultural Food Chemistry 50: 2076-2082. DOI: 10.1021/jf011074c

Department of Fertilizers, Government of India (2016) Import of Fertilizer. Available on Web: http://fert.nic.in/node/3808. access on 19th July, 2016

FAO-Food and Agriculture Organization (2014) FAOSTAT Debase. FAO, ROME. Available on Website:www.faostat.fao.org, accessed on 1st August, 2016.

Gardner FP, Pearce RB, Mithcell RL (1985) Carbon fixation by crop canopies. Ames: Iowa State University Press. pp. 3157. 
Gould WA (1999) Specific gravity of potatoes. In: Potato Production, Processing and Technology. CTI Publications Inc., Maryland, USA. pp. 51-72.

Hamouz K, Lachman J, Dvořák P, Orsák M, Hejtmánková K, Čížek M (2009) Effect of selected factors on the content of ascorbic acid in potatoes with different tuber flesh colour. Plant Soil Environment 55: 281-287.

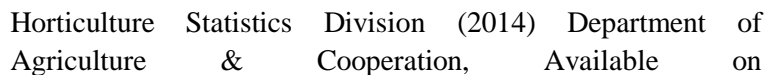
http://www.agricoop.nic.in/Admin_Agricoop/Uploaded_File/I CAR_5.pdf Government of India accessed on 1st August, 2016.

Kadian MS, Ilangantileke S, Arif M, Hossain M, Roder W, Sakla BM, Singh SV, Farooq K. Mazeen ACM (2007) Status of potato seed systems in South-West Asia (SWA). Potato Journal 34: 25-30.

Khan, M Z, Akhtar ME, Mahmood-ul-Hassan M, Mahmood MM, Safdar MN (2012) Potato tuber yield and quality as affected by rates and sources of potassium fertilizer. Journal of Plant Nutrition 35: 664-677. DOI: 10.1080/01904167.2012.653072

Kumar A, Tripathi HR, Yadav RA, Yadav DS (2008) Diversification of rice (Oryza sativa)-wheat (Triticum aestivum) cropping system for sustainable production in eastern Uttar Pradesh. Indian Journal of Agronomy 53: 18-21.

Kumar P, Pandey SK, Singh BP, Singh SV, Kumar D (2007) Influence of source and time of potassium application on potato growth, yield, economics and crisp quality. Potato Research 50: 1-13.

Manolov, I, Neshev N, Chalova V, Yordanova N (2015) Influence of potassium fertilizer source on potato yield and quality. Available on http://sa.agr.hr, 363, 363.

Moinuddin, Singh, K, Bansal SK, Pasricha NS (2004) Influence of graded levels of potassium fertilizer on growth, yield, and economic parameters of potato. Journal of Plant Nutrition 27: 239-259. DOI: 0.1081/PLN-120027652.

Mozumder M, Banerje, H, Ray K, Paul T (2014) Evaluation of potato (Solanum tuberosum) cultivars for productivity, N requirement and eco-friendly indices under different nitrogen levels. Indian Journal of Agronomy 59: 327-335.
Nelson N (1944) A photometric adaptation of the Somogyi method for the determination of glucose. Journal of Biological Chemistry 153: 375-80.

Nikolova M, Blagoeva V (2000) Yield and Quality of Potato as Affected by use of Potash. Leaflet, International Potash Institute.

Panagiotopoulos LI (1995) Fertilization of potato crops. Agriculture-Cattle Breeding 9: 227-231.

Rathore SS, Chaudhary DR, Vaisya LK, Shekhawat K, Bhatt BP (2014) Schoenite and potassium sulphate: Indigenous potassic fertilizer for rainfed groundnut (Arachis hypogaea L.). Indian Journal of Traditional Knowledge 13: 222-226.

Sarkar D, Mandal B, Kundu MC (2007) Increasing use efficiency of boron fertilizers by rescheduling the time and method of application for crops in India. Plant and Soil 301: 77-85. DOI:10.1007/s11104-007-9423-1

Sharma RC, Sud KC, Sood MC (1998). Potassium needs for high yield and quality of potato in Himachal Pradesh. In: Use of Potassium in Himachal Pradesh, Potash and Phosphate Institute of Canada, Gurgaon, pp. 44-57.

Singh JP, Marwha JS, Grewal JS (1996). Effect of sources and levels of potassium on potato yield, quality and storage behavior. Journal of Indian Potato Association 23: 153-156.

Smith O (1987) Effect of cultural and environmental conditions on potatoes for processing. In: Talburt WF, Smith O (Eds.), 4th edn. Potato processing. Van Nostrand Reinhold Company, New York, pp. 73-147.

Toolangi TK (1995) Potatoes: factors affecting dry matter. Agriculture Notes, April 1995, State of Victoria, Department of Primary Industries, Victoria, USA. pp. 1-3.

Watson DJ (1947) Comparative physiological studies on the growth of field crops: I. Variation in net assimilation rate and leaf area between species and varieties, and within and between years. Annals of Botany 11: 41-76.

Zorb C, Senbayram M, Peiter E (2014) Potassium in agriculture - Status and perspectives. Journal of Plant Physiology 171: 656-669. DOI: 10.1016/j.jplph.2013.08.008. 